\title{
Percutaneous vertebroplasty:can it be an alternative treatment option for thoracolumbar burst fractures?
}

\author{
Young Hwan Choi ${ }^{1}$, Young Hun Pee ${ }^{1}$, Il-Tae Jang ${ }^{2}$ \\ ${ }^{1}$ Department of Neurosurgery, Nanoori Hospital Jooan, Incheon, Korea \\ ${ }^{2}$ Department of Neurosurgery, Nanoori Hospital Gangnam, Seoul, Korea
}

Objective: The incidence of thoracolumbar fractures is increasing in modern society concomitantly with population aging. The role of percutaneous vertebroplasty as a possible treatment option for thoracolumbar fractures remains unclear.

Methods: In this study, we retrospectively reviewed clinical and radiological factors, including sex, age, bone mineral density, mechanisms of injury, fracture levels, and the Magerl classification in 101 patients (108 vertebral levels) with thoracolumbar burst fractures that were treated with vertebroplasty.

Results: After vertebroplasty, the mean visual analog scale score immediately decreased from 6.3 to 2.7 . The average preoperative, postoperative, and follow-up values of the sagittal index were $9.5^{\circ}, 7.6^{\circ}$, and $10.0^{\circ}$, respectively. The average preoperative, postoperative, and follow-up canal compromise values were $19.3 \%, 12.7 \%$, and $18.3 \%$, respectively. No severe complications were associated with the procedure. Canal compromise and the sagittal index remained stable during the immediate postoperative period and even after 3 months postoperatively.

Conclusion: Vertebroplasty is a promising treatment choice for burst fractures, even in symptomatic patients.

Keywords: Spinal fractures; Vertebroplasty; Thoracic vertebrae; Lumbar vertebrae

\section{Introduction}

With the increase in the elderly population in modern society, there has been an increase in thoracolumbar fractures. Management of thoracolumbar fractures is a socioeconomic burden. Additionally, the elderly often have preexisting comorbidities, such as osteoporosis, which increase osseous nonunion risk [1]. Therefore, surgical procedures, such as corpectomy and fusion, can be complicated.

Burst fractures are classified according to the Magerl classification. Magerl type A3 is used to define thoracolumbar burst fractures. It is further classified into three types: A3.1, incomplete burst fracture; A3.2, burst split fracture; and A3.3, complete burst

\footnotetext{
Received: November 15, 2020 Revised: November 30, 2020

Accepted: December 5, 2020

Corresponding Author: Il-Tae Jang, MD, PhD

Department of Neurosurgery, Nanoori Hospital Gangnam, 731 Eonju-ro,

Gangnam-gu, Seoul 06048, Korea

E-mail: nanoori_research@naver.com
}

fracture [2].

Treatment options for managing Magerl type A3 fractures are still controversial and vary from conservative to operative treatment [3]. Less invasive treatment is needed in patients who do not respond well to conservative treatment. Therefore, In this study, the role of percutaneous vertebroplasty in the thoracolumbar burst fracture treatment was evaluated.

Sagittal index and canal compromise were used as measurement methods that could reflect the fracture shape while being related to the degree of neurological damage [4]. Successful treatment of osteoporotic vertebral compression and burst fractures without neural deficit by percutaneous transpedicular vertebroplasty has been reported previously $[1,5]$.

In our study, we also included patients with neurological symptoms. Therefore, this study will provide sufficient evidence in favor of vertebroplasty as a less invasive treatment option for thoracolumbar burst fractures. 


\section{Material and Method}

\section{Ethics statement}

This study was reviewed and approved by the Institutional Review Board (IRB No: NR-IRB 2020-029). Informed consent was obtained from all individual participants included in this study.

\section{Patient population}

Between December 2015 and December 2019, percutaneous vertebroplasty was performed in 101 patients with 108 levels of thoracolumbar burst fracture (Margerl type A3). The selected patients were limited to those who had acute back/low back pain without neurological deficits, and their diagnosis was confirmed by magnetic resonance imaging (MRI). Vertebral body fractures were classified according to the Magerl classification. Demographic data were collected, including age, sex, fracture level, visual analog scale (VAS), and cause of the accident. Thirty-one patients had neurologic symptoms, including radiating pain or numbness in the buttock and leg. There was no motor weakness in the selected patients. Patients with neurological deficits, such as muscle weakness, paresthesia, and cauda equina syndrome, were treated surgically and excluded from this study.

\section{Imaging study}

Preoperative radiographs, MRI, and dual-energy X-ray absorptiometry were performed for all patients. Immediate postoperative computed tomography (CT) or MRI were performed on all patients after the surgery. Follow-up CT or MRI were performed in 53 patients at least three months after the surgery. Sagittal index and canal compromise of the fractured vertebrae were analyzed during the preoperative, postoperative, and follow-up stages.

Spinal canal compromise was measured using CT or MRI. The mid-sagittal diameter of the spinal canal at the level of the fracture was measured. Un-injured adjacent vertebral levels above and below the injured vertebra were used for estimating the mid-sagittal diameter of the original spinal canal. The percentage of spinal canal compromise was calculated using the formula: ([1 - mid-sagittal diameter of the spinal canal at the level of injury/mean of the mid-sagittal diameters of the spinal canal one segment above and below the level of injury] x 100) [6].

The sagittal index was defined to help in the assessment of the segmental deformity at the level of the injury. Segmental kyphosis was measured as the lateral Cobb angle and was evaluated in terms of the sagittal index at the preoperative, postoperative, and follow-up stages. The baseline sagittal curve/level was estimated as $5^{\circ}$ in thoracic spine segments, $0^{\circ}$ at the thoracolumbar junction, and $-10^{\circ}$ in the lumbar spine. The sagittal index was then defined as the local kyphotic deformity minus the baseline sagittal curve at the level of the injury $[7,8]$.

\section{Surgical technique}

Percutaneous vertebroplasty was performed under C-arm monitoring. An hour before the surgery, antibiotics were administered to the patient. The patient was in a prone position and was draped with a sterile cloth. One percent lidocaine was used for local anesthesia. After that, a Jamshidi needle was advanced to the fractured vertebral body via the pedicle. Polymethyl methacrylate (PMMA) powder was mixed, and the viscosity of the paste was made similar to that of toothpaste to prevent cement leakage. The mixture was placed in a $1 \mathrm{~mL}$ syringe, which was used as an injector. C-arm fluoroscopy was used in lateral projection during cement injection.

\section{Results}

The study included 16 males and 85 females. The mean age of the patients at the time of vertebroplasty was 72 years in males and 75 years in females. The mean bone mineral density (BMD) among males and females was -2.1 and -3.0, respectively. A statistically significant difference was observed between males and females. Osteoporosis was observed in 65 patients. The mechanisms of injury included slipping in 49 patients (48.5\%), unknown in 35 patients (34.7\%), and sprain in 17 patients (16.8\%) (Table 1$)$.

The number of fracture levels according to Magerl classification was as follows: A3.1, 87 levels; A3.2, 11; A3.3, 10 (Table 2). The most commonly involved vertebra was $\mathrm{L} 1$, and the most common type of burst fracture according to the Magerl classification was type A3.1 (Table 2). The average preoperative and postoperative VAS scores were 6.3 and 2.7, respectively. Immediate pain reduction was statistically confirmed (Table 3 ).

Radiological analysis was performed using the sagittal index and canal compromise. The average preoperative, postoperative, and follow-up sagittal index values were $9.5^{\circ}, 7.6^{\circ}$, and $10.0^{\circ}$, respectively (Fig. 1). The average preoperative, postoperative, and follow-up

Table 1. Age, BMD, and cause of accident according to sex

\begin{tabular}{lccc}
\hline Variable & Female $(n=85)$ & Male $(n=16)$ & P-value \\
\hline Age $(y r)$ & $75.3 \pm 8.9$ & $72.2 \pm 9.3$ & 0.216 \\
BMD & $-3.0 \pm 1.1$ & $-2.1 \pm 1.5$ & $0.005^{*}$ \\
Cause & & & 0.945 \\
$\quad$ Unknown & $30(35.3)$ & $5(31.2)$ & \\
$\quad$ Sprain & $14(16.5)$ & $3(18.8)$ & \\
$\quad$ Slip down & $41(48.2)$ & $8(50.0)$ & \\
\hline
\end{tabular}

Values are presented as mean \pm standard deviation or number (\%). $\mathrm{BMD}$, bone mineral density.

*Statistically significant differences $(\mathrm{P}<0.05)$. 
canal compromise values were $19.3 \%, 12.7 \%$, and $18.3 \%$, respectively (Fig. 2).

There were no serious complications associated with the procedure. A small amount of PMMA leakage into the spinal canal was observed in two cases, however, these patients had no symptoms. None of the patients required surgery due to neurological or radiographic deterioration after vertebroplasty.

Table 2. The number of fracture levels according to the Magerl classification

\begin{tabular}{ccccc}
\hline Variable & A3.1 $(n=87)$ & A3.2 $(n=11)$ & A3.3 $(n=10)$ & P-value \\
\hline Level & & & & 0.211 \\
L1 & $27(31.0)$ & $4(36.4)$ & $1(10)$ & \\
L2 & $17(19.5)$ & $1(9.1)$ & $2(20)$ & \\
L3 & $7(8.0)$ & 0 & $2(20)$ & \\
L4 & $3(3.4)$ & $1(9.1)$ & $1(10)$ & \\
T10 & $1(1.1)$ & 0 & $1(10)$ & \\
T11 & $6(6.9)$ & $1(9.1)$ & 0 & \\
T12 & $21(24.1)$ & $3(27.3)$ & $2(20)$ & \\
T5 & $2(2.3)$ & 0 & 0 & \\
T7 & $1(1.1)$ & 0 & $1(10)$ & \\
T8 & 0 & $1(9.1)$ & 0 & \\
T9 & $2(2.3)$ & 0 & 0 & \\
\hline
\end{tabular}

Values are presented as number (\%).

$L$, lumbar spine vertebrae; $T$, thoracic spine vertebrae.

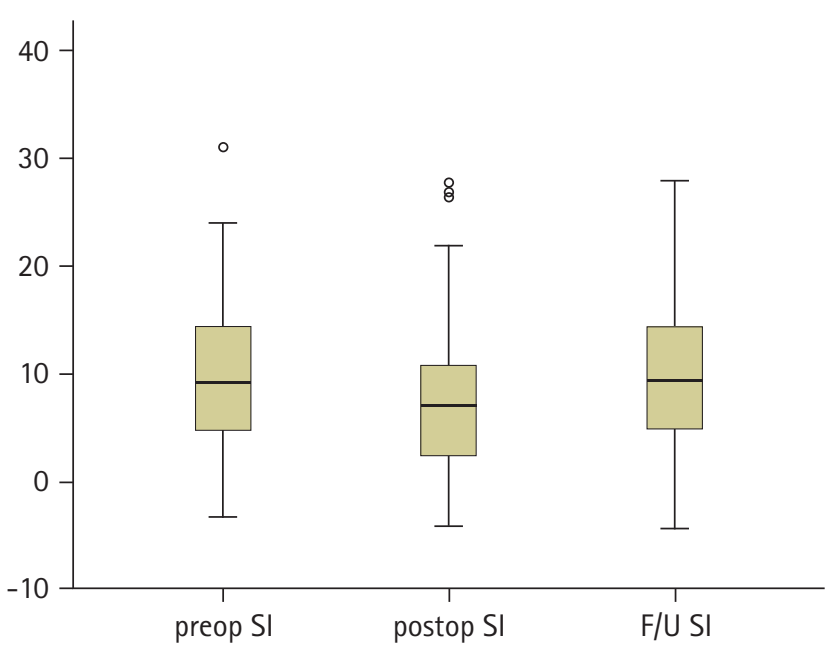

Fig. 1. Radiological analysis of the sagittal index (SI) of fractured vertebrae preoperatively (preop), postoperatively (postop), and at follow-up (F/U). ○, outliers.

\section{Discussion}

Percutaneous vertebroplasty can be differentiated from conservative treatment and surgical treatment modalities. In older patients, long-term conservative treatment decreases muscle strength and increases the incidence of thromboembolism, pulmonary complications, and depression [9]. Invasive treatments such as corpectomy have a high complication rate and are burdensome [1]. Vertebroplasty is a less invasive method with less overall treatment burden. Additionally, it is possible to achieve the therapeutic goal of pain reduction and structural stability with vertebroplasty. In particular, it has been performed reluctantly because of the risk of canal compromise progression and cement leakage [10]. In this study, these risks did not seem to be significant.

In the present study, an immediate postoperative VAS reduction was observed in the patients. This may be because of the effect of fracture stabilization at the fracture site and thermal or chemical ablation of PMMA on the nerve endings [11]. Reduction of VAS also signifies an early return to daily life activities and work.

The radiological results showed improvement in the sagittal in dex and canal compromise after the procedure and showed that this effect is maintained even during the follow-up period. The following reason could explain this: the fractured bone was reduced while the patient was in a prone position, and the cement penetrat-

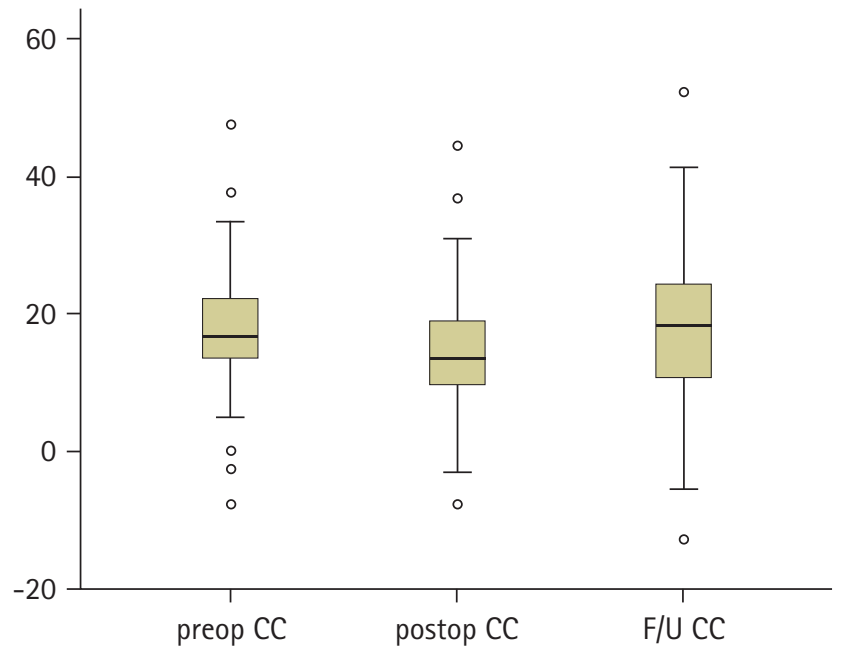

Fig. 2. Radiological analysis of canal compromise (CC) of fractured vertebrae preoperatively (preop), postoperatively (postop), and at follow-up (F/U). ○, outliers.

Table 3. The pre- and postoperative VAS

\begin{tabular}{|c|c|c|c|c|c|c|c|}
\hline \multirow{2}{*}{ Variable } & \multicolumn{4}{|c|}{ Paired difference } & \multirow{2}{*}{$\mathrm{t}$} & \multirow{2}{*}{ df } & \multirow{2}{*}{$\begin{array}{l}\text { Significance } \\
\text { (2-tailed) }\end{array}$} \\
\hline & Mean & SD & SE & $95 \% \mathrm{Cl}$ & & & \\
\hline Preoperative VAS-postoperative VAS & 3.63 & 1.655 & 0.159 & $3.314-3.945$ & 22.787 & 107 & 0.000 \\
\hline
\end{tabular}

VAS, visual analog scale; SD, standard deviation; $\mathrm{SE}$, standard error of the mean; $\mathrm{Cl}$, confidence interval. 
ed the fractured bone. Over time, the reduction in cement-free areas may have subsided but might not have progressed beyond the procedural area.

Vertebroplasty provides structural stability by increasing the strength of fractured bones and providing anterior support by holding the immediately fractured bone fragments tightly [10].

\section{Conflicts of interest}

No potential conflict of interest relevant to this article was reported.

\section{REFERENCES}

1. Purvis TE, De la Garza Ramos R, Sankey EW, Karikari IO, Goodwin CR, Sciubba DM. In-hospital medical complications after non-operative and operative treatment of thoracolumbar fractures in patients over 75 years of age. J Clin Neurosci 2018; 50:83-7.

2. Magerl F, Aebi M, Gertzbein SD, Harms J, Nazarian S. A comprehensive classification of thoracic and lumbar injuries. Eur Spine J 1994;3:184-201.

3. Shen J, Xu L, Zhang B, Hu Z. Risk factors for the failure of spinal burst fractures treated conservatively according to the thoracolumbar injury classification and severity score (TLICS): a retrospective cohort trial. PLoS One 2015;10:e0135735.

4. Yüksel MO, Gurbuz MS, Gok S, Karaarslan N, Is M, Berkman
MZ. The association between sagittal index, canal compromise, loss of vertebral body height, and severity of spinal cord injury in thoracolumbar burst fractures. J Neurosci Rural Pract 2016; 7(Suppl 1):S57-61.

5. Nakano M, Hirano N, Matsuura K, et al. Percutaneous transpedicular vertebroplasty with calcium phosphate cement in the treatment of osteoporotic vertebral compression and burst fractures. J Neurosurg 2002;97(3 Suppl):287-93.

6. Mohanty SP, Bhat NS, Abraham R, Ishwara Keerthi C. Neurological deficit and canal compromise in thoracolumbar and lumbar burst fractures. J Orthop Surg (Hong Kong) 2008; 16:20-3.

7. Farcy JP, Weidenbaum M, Glassman SD. Sagittal index in management of thoracolumbar burst fractures. Spine (Phila Pa 1976) 1990;15:958-65.

8. Jiang SD, Wu QZ, Lan SH, Dai LY. Reliability of the measurement of thoracolumbar burst fracture kyphosis with Cobb angle, Gardner angle, and sagittal index. Arch Orthop Trauma Surg 2012;132:221-5.

9. Hartmann F, Gercek E, Leiner L, Rommens PM. Kyphoplasty as an alternative treatment of traumatic thoracolumbar burst fractures Magerl type A3. Injury 2012;43:409-15.

10. Wang H, Zhang Z, Liu Y, Jiang W. Percutaneous kyphoplasty for the treatment of very severe osteoporotic vertebral compression fractures with spinal canal compromise.J Orthop Surg Res 2018;13:13.

11. Wood KB, Li W, Lebl DR, Ploumis A. Management of thoracolumbar spine fractures. Spine J 2014;14:145-64. 